\title{
On Some Fractional Hardy-Hilbert's Integral Inequalities
}

\author{
Atta A.K. Abu Hany ${ }^{* 1}$, Mohammed S. El-Khatib ${ }^{2}$, Abbas K. Shourrab ${ }^{3}$ \\ 1,2,3 Department of Mathematics, Al-Azhar Universiry-Gaza, Palestine \\ ${ }^{1}$ attahany@gmail.com, ${ }^{2}$ msmkhatib@yahoo.com, ${ }^{3}$ abbasshourab@gmail.com
}

Abstract. We focus our attention in this article on some recent results regarding Hardy-Hilbert's inequalities. We derive an equivalent form using katugampola Fractional Calculus and introduce new analogs to some Hardy-Hilbert's type inequality. Several special cases are also given.

Keywords: Katugampola fractional integral; Katugampola fractional derivative; Hardy-Hilbert's inequality; Equivalent forms.

2010 MSC No: 26D15, 26A33

\section{Introduction}

Katugampola Fractional Calculus is a new Fractional Calculus introduced and studied by Katugampola in the year 2014 which is more suited to the approximation of classical calculus. In [6], the author gave the definitions of Katugampola fractional derivative and Katugampola fractional integral as follows:

Definition $1.1[6]$ Let $f:[0, \infty) \rightarrow R$ and $t>0$. Then the "Katugampola fractional derivative" of $f$ of order $\alpha$ is defined by

$$
D^{\alpha}(f)(t)=\lim _{\varepsilon \rightarrow 0} \frac{f\left(t e^{\varepsilon t^{-\alpha}}\right)-f(t)}{\varepsilon},
$$

for $t>0$ and $\alpha \in(0,1]$. If $f$ is $\alpha$-differentiable in some $(0, a), a>0$ and $\lim _{t \rightarrow 0^{+}} D^{\alpha}(f)(t)$ exists, then $D^{\alpha}(f)(0)=$ $\lim _{t \rightarrow 0^{+}} D^{\alpha}(f)(t)$.

If $f$ is differentiable, then we have $D^{\alpha}(f)(t)=t^{1-\alpha} \frac{d f}{d t}$.

Definition 1.2 (Katugampola Fractional integral) [6] Let $a \geq 0$ and $t \geq a$. Also, let $f$ be a function defined on $(a, t]$ and $\alpha \in R$. Then, the $\alpha$-fractional integral of $f$ is defined by,

$$
I_{a}^{\alpha}(f)(t)=\int_{a}^{t} \frac{f(x)}{x^{1-\alpha}} d x
$$

if the Riemann improper integral exists.

Definition 1.3 Let $\alpha \in(0,1]$ and $0 \leq a<b$. A function $f:[a, b] \rightarrow R$ is $\alpha$-fractional integrable on $[a, b]$

\footnotetext{
${ }^{*}$ Corresponding author. Atta A.K. Abu Hany ${ }^{1}$ attahany@gmail.com
} 
if the integral

$$
\int_{a}^{b} f(t) d_{\alpha} t:=\int_{a}^{b} f(t) t^{\alpha-1} d t
$$

exists.

The well-known Hilbert's inequality and its equivalent form are given as :

Theorem A. [5] If $f$ and $g \in L^{2}[0, \infty)$, then the following inequalities hold and are equivalent

$$
\int_{0}^{\infty} \int_{0}^{\infty} \frac{f(x) g(x)}{x+y} d x d y \leq \pi\left(\int_{0}^{\infty} f^{2}(x) d x\right)^{1 / 2}\left(\int_{0}^{\infty} g^{2}(y) d y\right)^{1 / 2},
$$

and

$$
\int_{0}^{\infty}\left(\int_{0}^{\infty} \frac{f(x)}{x+y} d x\right)^{2} d y \leq \pi^{2} \int_{0}^{\infty} f^{2}(x) d x,
$$

where $\pi$ and $\pi^{2}$ are optimal .

Hilbert's integral inequality (4) had been generalized by Hardy- Riesz (see[3]) in 1925 as the following result. If $f, g$ are nonnegative functions such that $0<\int_{0}^{\infty} f^{p}(x) d x<\infty$, and

$0<\int_{0}^{\infty} g^{q}(x) d x<\infty$, where $\frac{1}{p}+\frac{1}{q}=1$, then

$$
\int_{0}^{\infty} \int_{0}^{\infty} \frac{f(x) g(x)}{x+y} d x d y \leq \pi \csc (\pi / p)\left(\int_{0}^{\infty} f^{p}(x) d x\right)^{1 / p}\left(\int_{0}^{\infty} g^{q}(y) d y\right)^{1 / q},
$$

where the constant factor $\pi \csc (\pi / p)$ is the best possible. When $p=q=2$, inequality (6) is reduced to (4).

In recent years, a number of mathematicians had given lots of generalizations of these inequalities. We mention here some of these contributions in this direction :

Li et al. [8] have proved the following Hardy- Hilbert's type inequality using the hypotheses of (4):

$$
\int_{0}^{\infty} \int_{0}^{\infty} \frac{f(x) g(x)}{x+y+\max \{x, y\}} d x d y<c\left(\int_{0}^{\infty} f^{2}(x) d x\right)^{1 / 2}\left(\int_{0}^{\infty} g^{2}(y) d y\right)^{1 / 2},
$$

where the constant factor $c=\sqrt{2}\left(\pi-2-\tan ^{-1} \sqrt{2}\right)=1.7408 \ldots$ is the best possible.

Y. Li, Y. Qian, and B. He [9] deduced the following result :

Theorem B. If $f, g \geq 0,0<\int_{0}^{\infty} f^{2}(x) d x<\infty$, and $0<\int_{0}^{\infty} g^{2}(x) d x<\infty$, then one has

$$
\int_{0}^{\infty} \int_{0}^{\infty} \frac{|\ln x-\ln y|}{x+y+|x-y|} f(x) g(y) d x d y<4\left(\int_{0}^{\infty} f^{2}(x) d x\right)^{1 / 2}\left(\int_{0}^{\infty} g^{2}(x) d x\right)^{1 / 2},
$$

where the constant factors 4 is the best possible.

More and more results regarding this direction on Hilbert's type inequalities can be found for example in $[4$, $7,10]$.

\section{Main Results}

The main objective of this paper is to build a new equivalent form inequality depending on some particular new results given in article [1]. We also introduce new analogs of fractional form to some Hardy-Hilbert's type inequality.

In this section we shall prove some lemmas which play crucial roles in proving our main results. The following one is useful in establishing our results. 
Lemma $2.1[1]$ Let $a, b, c$ be three non-negative real numbers and $\alpha \in(0,1]$. Then we have the following equations

$$
\begin{aligned}
\int_{0}^{\infty} \frac{\alpha^{c+1}|\ln x-\ln y|^{c}}{a x^{\alpha}+b y^{\alpha}+\left|x^{\alpha}-y^{\alpha}\right|}\left(\frac{x}{y}\right)^{\alpha / 2} d_{\alpha} y & =\int_{0}^{\infty} \frac{\alpha^{c+1}|\ln x-\ln y|^{c}}{a x^{\alpha}+b y^{\alpha}+\left|x^{\alpha}-y^{\alpha}\right|}\left(\frac{y}{x}\right)^{\alpha / 2} d_{\alpha} x \\
& =\int_{0}^{\alpha^{\frac{1}{\alpha}}} \frac{2^{c+1} \alpha^{2}|\alpha \ln (t)-\ln \alpha|^{c}}{\alpha^{2}(a+1)+t^{2 \alpha}(b-1)} d_{\alpha} t \\
& +\int_{0}^{\alpha^{\frac{1}{\alpha}}} \frac{2^{c+1} \alpha^{2}|\alpha \ln (t)-\ln \alpha|^{c}}{\alpha^{2}(b+1)+t^{2 \alpha}(a-1)} d_{\alpha} t \\
& =A
\end{aligned}
$$

where $A:=A(a, b, c) \in[0, \infty]$.

The results stated in the following theorem is under consideration.

Theorem 2.1 [1] Let $f$ and $g$ be two $\alpha$-fractional integrable functions on $[0, \infty)$ such that

$$
0<\int_{0}^{\infty} f^{2}(x) d_{\alpha} x<\infty, 0<\int_{0}^{\infty} g^{2}(x) d_{\alpha} x<\infty
$$

then we have

$$
\begin{gathered}
\int_{0}^{\infty} \int_{0}^{\infty} \frac{\alpha^{c+1}|\ln x-\ln y|^{c}}{a x^{\alpha}+b y^{\alpha}+\left|x^{\alpha}-y^{\alpha}\right|} f(x) g(y) d_{\alpha} x d_{\alpha} y \\
<A\left(\int_{0}^{\infty} f^{2}(x) d_{\alpha} x\right)^{1 / 2}\left(\int_{0}^{\infty} g^{2}(y) d_{\alpha} y\right)^{1 / 2},
\end{gathered}
$$

where $A$ is defined in lemma 2.1 and it is the best possible constant.

In the following theorem, we introduce an equivalent form to inequality (9).

Theorem 2.2 Suppose $f \geq 0, \alpha \in(0,1]$, and $0<\int_{0}^{\infty} f^{2}(x) d_{\alpha} x<\infty$, then

$$
\int_{0}^{\infty}\left[\int_{0}^{\infty} \frac{\alpha^{c+1}|\ln x-\ln y|^{c}}{a x^{\alpha}+b y^{\alpha}+\left|x^{\alpha}-y^{\alpha}\right|} f(x) d_{\alpha} x\right]^{2} d_{\alpha} y=A^{2} \int_{0}^{\infty} f^{2}(x) d_{\alpha} x,
$$

where $A$ is defined in Lemma 2.1. Furthermore, Inequality (10) is equivalent to (9).

Proof: Let

$$
I=\int_{0}^{\infty}\left[\int_{0}^{\infty} \frac{\alpha^{c+1}|\ln x-\ln y|^{c}}{a x^{\alpha}+b y^{\alpha}+\left|x^{\alpha}-y^{\alpha}\right|} f(x) d_{\alpha} x\right]^{2} d_{\alpha} y
$$

Setting $x^{\alpha}=\frac{(y z)^{\alpha}}{\alpha}, d_{\alpha} x=\frac{y^{\alpha}}{\alpha} d_{\alpha} z$, then we get

$$
I=\int_{0}^{\infty}\left[\int_{0}^{\infty} \frac{\alpha^{c+1}|\ln z-\ln \alpha|^{c}}{a z^{\alpha}+b \alpha+\left|z^{\alpha}-\alpha\right|} f\left(\frac{y z}{\alpha^{\frac{1}{\alpha}}}\right) d_{\alpha} z\right]^{2} d_{\alpha} y
$$

By Minkowski's inequality for integrals,

$$
I \leq\left(\int_{0}^{\infty}\left[\left(\int_{0}^{\infty} \frac{\alpha^{c+1}|\ln z-\ln \alpha|^{c}}{a z^{\alpha}+b \alpha+\left|z^{\alpha}-\alpha\right|} f\left(\frac{y z}{\alpha^{\frac{1}{\alpha}}}\right)\right)^{2} d_{\alpha} y\right]^{1 / 2} d_{\alpha} z\right)^{2}
$$




$$
I \leq\left(\int_{0}^{\infty} \frac{\alpha^{c+1}|\ln z-\ln \alpha|^{c}}{a z^{\alpha}+b \alpha+\left|z^{\alpha}-\alpha\right|}\left[\left(\int_{0}^{\infty} f^{2}\left(\frac{y z}{\alpha^{\frac{1}{\alpha}}}\right)\right) d_{\alpha} y\right]^{1 / 2} d_{\alpha} z\right)^{2} .
$$

Setting $y^{\alpha}=\frac{\alpha u^{\alpha}}{z^{\alpha}}, d_{\alpha} y=\frac{\alpha}{z^{\alpha}} d_{\alpha} u$, then by Fubini's Theorem, we obtain

$$
\begin{aligned}
I & \leq\left(\int_{0}^{\infty} \frac{\alpha^{c+1}|\ln z-\ln \alpha|^{c} \alpha^{1 / 2} z^{-\alpha / 2}}{a z^{\alpha}+b \alpha+\left|z^{\alpha}-\alpha\right|} d_{\alpha} z\left[\left(\int_{0}^{\infty} f^{2}(u)\right) d_{\alpha} u\right]^{1 / 2}\right)^{2}, \\
I & \leq\left(\int_{0}^{\infty} \frac{\alpha^{c+1}|\ln z-\ln \alpha|^{c} \alpha^{1 / 2} z^{-\alpha / 2}}{a z^{\alpha}+b \alpha+\left|z^{\alpha}-\alpha\right|} d_{\alpha} z\right)^{2} \int_{0}^{\infty} f^{2}(u) d_{\alpha} \\
& =A^{2} \int_{0}^{\infty} f^{2}(x) d_{\alpha} x .
\end{aligned}
$$

Thus, Inequality (10) holds.

Now, we prove that Inequality (10) is equivalent to (9). Suppose that Inequality (9) holds, and let

$$
g(y)=\int_{0}^{\infty} \frac{\alpha^{c+1}|\ln x-\ln y|^{c}}{a x^{\alpha}+b y^{\alpha}+\left|x^{\alpha}-y^{\alpha}\right|} f(x) d_{\alpha} x .
$$

Hence

$$
0<\int_{0}^{\infty} g^{2}(y) d_{\alpha} y=\int_{0}^{\infty}\left(\int_{0}^{\infty} \frac{\alpha^{c+1}|\ln x-\ln y|^{c}}{a x^{\alpha}+b y^{\alpha}+\left|x^{\alpha}-y^{\alpha}\right|} f(x) d_{\alpha} x\right) g(y) d_{\alpha} y .
$$

By Fubini's Theorem and Inequalities (9),

$$
\begin{aligned}
\int_{0}^{\infty} g^{2}(y) d_{\alpha} y= & \int_{0}^{\infty} \int_{0}^{\infty} \frac{\alpha^{c+1}|\ln x-\ln y|^{c}}{a x^{\alpha}+b y^{\alpha}+\left|x^{\alpha}-y^{\alpha}\right|} f(x) g(y) d_{\alpha} x d_{\alpha} y . \\
& <A\left(\int_{0}^{\infty} f^{2}(x) d_{\alpha} x\right)^{1 / 2}\left(\int_{0}^{\infty} g^{2}(y) d_{\alpha} y\right)^{1 / 2} .
\end{aligned}
$$

Notice that by Inequality (10), $g \in L^{2}$. So the last integral is finite, and hence

$$
\left(\int_{0}^{\infty} g^{2}(y) d_{\alpha} y\right)^{1 / 2} \leq A\left(\int_{0}^{\infty} f^{2}(x) d_{\alpha} x\right)^{1 / 2} .
$$

Thus

$$
\int_{0}^{\infty}\left(\int_{0}^{\infty} \frac{\alpha^{c+1}|\ln x-\ln y|^{c}}{a x^{\alpha}+b y^{\alpha}+\left|x^{\alpha}-y^{\alpha}\right|} f(x) d_{\alpha} x\right)^{2} d_{\alpha} y \leq A^{2} \int_{0}^{\infty} f^{2}(x) d_{\alpha} x .
$$

Conversly, if Inequality (10) holds, then

$$
\begin{aligned}
\int_{0}^{\infty} \int_{0}^{\infty} \frac{\alpha^{c+1}|\ln x-\ln y|^{c}}{a x^{\alpha}+b y^{\alpha}+\left|x^{\alpha}-y^{\alpha}\right|} f(x) g(y) d_{\alpha} x d_{\alpha} y \\
\quad=\int_{0}^{\infty}\left(\int_{0}^{\infty} \frac{\alpha^{c+1}|\ln x-\ln y|^{c}}{a x^{\alpha}+b y^{\alpha}+\left|x^{\alpha}-y^{\alpha}\right|} f(x) d_{\alpha} x\right) g(y) d_{\alpha} y
\end{aligned}
$$

By Cauchy - Schwarz inequality we get

$$
\int_{0}^{\infty}\left(\int_{0}^{\infty} \frac{\alpha^{c+1}|\ln x-\ln y|^{c}}{a x^{\alpha}+b y^{\alpha}+\left|x^{\alpha}-y^{\alpha}\right|} f(x) d_{\alpha} x\right) g(y) d_{\alpha} y
$$




$$
\begin{aligned}
& \leq\left(\int_{0}^{\infty}\left[\int_{0}^{\infty} \frac{\alpha^{c+1}|\ln x-\ln y|^{c}}{a x^{\alpha}+b y^{\alpha}+\left|x^{\alpha}-y^{\alpha}\right|} f(x) d_{\alpha} x\right]^{2} d_{\alpha} y\right)^{1 / 2}\left(\int_{0}^{\infty} g^{2}(y) d_{\alpha} y\right)^{1 / 2} \\
& \leq A\left(\int_{0}^{\infty} f^{2}(x) d_{\alpha} x\right)^{1 / 2}\left(\int_{0}^{\infty} g^{2}(y) d_{\alpha} y\right)^{1 / 2} .
\end{aligned}
$$

The following lemma is being used in establishing the rest of main results.

Lemma 2.2 Let $f$ be a nonnegative integrable function, and

$$
F(x)=\int_{0}^{x^{\alpha} / \alpha} f\left((\alpha t)^{\frac{1}{\alpha}}\right) d_{\alpha} t
$$

then

$$
\int_{0}^{\infty}\left(\frac{\alpha F(x)}{x^{\alpha}}\right)^{p} d_{\alpha} x<\left(\frac{p}{p-1}\right)^{p} \int_{0}^{\infty} f^{p}(x) d_{\alpha} x, \alpha \in(0,1], p>1 .
$$

Proof: If $0<\xi<X$, we have

$$
\begin{aligned}
I & =\int_{\xi}^{X}\left(\frac{\alpha F(x)}{x^{\alpha}}\right)^{p} d_{\alpha} x \\
& =\frac{1}{1-p} \int_{\xi}^{X} F^{p}(x) D_{\alpha}\left(\frac{x^{\alpha-\alpha p}}{\alpha^{1-p}}\right) d_{\alpha} x \\
& =\frac{1}{1-p} \int_{\xi}^{X} F^{p}(x) x^{1-\alpha} D\left(\frac{x^{\alpha-\alpha p}}{\alpha^{1-p}}\right) x^{\alpha-1} d x \\
& =\frac{1}{1-p} \int_{\xi}^{X} F^{p}(x) D\left(\frac{x^{\alpha-\alpha p}}{\alpha^{1-p}}\right) d x .
\end{aligned}
$$

Using the integral by parts we have ,

$$
\begin{aligned}
I & =\frac{1}{1-p}\left[\left.\frac{x^{\alpha-\alpha p}}{\alpha^{1-p}} F^{p}(x)\right|_{\xi} ^{X}-\frac{p}{\alpha^{1-p}} \int_{\xi}^{X} x^{\alpha-\alpha p} F^{p-1}(x) D(F(x)) d x\right] \\
& =\frac{1}{p-1}\left[\frac{\xi^{\alpha-\alpha p}}{\alpha^{1-p}} F^{p}(\xi)-\frac{X^{\alpha-\alpha p}}{\alpha^{1-p}} F^{p}(X)+\frac{p}{\alpha^{1-p}} \int_{\xi}^{X} x^{\alpha-\alpha p} F^{p-1}(x) D\left(\int_{0}^{x^{\alpha} / \alpha} f\left((\alpha t)^{\frac{1}{\alpha}}\right) d_{\alpha} t\right) d x\right] \\
& =\frac{1}{p-1}\left[\frac{\xi^{\alpha-\alpha p}}{\alpha^{1-p}} F^{p}(\xi)-\frac{X^{\alpha-\alpha p}}{\alpha^{1-p}} F^{p}(X)+\frac{p}{\alpha^{1-p}} \int_{\xi}^{X} x^{\alpha-\alpha p} F^{p-1}(x) f(x) x^{\alpha-1} d x\right] \\
& =\frac{1}{p-1}\left[\frac{\xi^{\alpha-\alpha p}}{\alpha^{1-p}} F^{p}(\xi)-\frac{X^{\alpha-\alpha p}}{\alpha^{1-p}} F^{p}(X)\right]+\frac{p}{p-1} \int_{\xi}^{X}\left(\frac{\alpha F(x)}{x^{\alpha}}\right)^{p-1} f(x) d_{\alpha} x .
\end{aligned}
$$

But $\xi^{\alpha-\alpha p} F^{p}(\xi) \rightarrow 0$ when $f(x)$ is integrable and $\xi \rightarrow 0$. Hence

$$
\begin{aligned}
\int_{0}^{X}\left(\frac{\alpha F(x)}{x^{\alpha}}\right)^{p} d_{\alpha} & \leq \frac{p}{p-1} \int_{0}^{X}\left(\frac{\alpha F(x)}{x^{\alpha}}\right)^{p-1} f(x) d_{\alpha} x \\
& \leq \frac{p}{p-1}\left\{\int_{0}^{X}\left(\frac{\alpha F(x)}{x^{\alpha}}\right)^{p} d_{\alpha} x\right\}^{p-1 / p}\left\{\int_{0}^{X} f^{p}(x) d_{\alpha} x\right\}^{1 / p} \cdot \text { (Holder inequality) }
\end{aligned}
$$


If $f$ is not nul in $(0, X)$, the left-hand side of $(12)$ is positive. Hence,

$$
\frac{\int_{0}^{X}\left(\frac{\alpha F(x)}{x^{\alpha}}\right)^{p} d_{\alpha} x}{\left\{\int_{0}^{X}\left(\frac{\alpha F(x)}{x^{\alpha}}\right)^{p} d_{\alpha} x\right\}^{p-1 / p}} \leq \frac{p}{p-1}\left\{\int_{0}^{X} f^{p}(x) d_{\alpha} x\right\}^{1 / p}
$$

take power $p$ for left-hand side, we have

$$
\int_{0}^{X}\left(\frac{\alpha F(x)}{x^{\alpha}}\right)^{p} d_{\alpha} x \leq\left(\frac{p}{p-1}\right)^{p} \int_{0}^{X} f^{p}(x) d_{\alpha} x
$$

and when make $X \rightarrow \infty$ we obtain (11), expect that " $<$ is replaced by " $\leq$.

In particular, the integral on the left-hand side of (11) is finite. It follows that all the integral (12) remains finite when $X$ is replaced by $\infty$, and that

$$
\begin{aligned}
\int_{0}^{\infty}\left(\frac{\alpha F(x)}{x^{\alpha}}\right)^{p} d_{\alpha} x & \leq \frac{p}{p-1} \int_{0}^{\infty}\left(\frac{\alpha F(x)}{x^{\alpha}}\right)^{p-1} f(x) d_{\alpha} x \\
& \leq \frac{p}{p-1}\left\{\int_{0}^{\infty}\left(\frac{\alpha F(x)}{x^{\alpha}}\right)^{p} d_{\alpha} x\right\}^{p-1 / p}\left\{\int_{0}^{\infty} f^{p}(x) d_{\alpha} x\right\}^{1 / p}
\end{aligned}
$$

The last sign of inequality may be replaced by "<" unless $\left(\frac{\alpha}{x^{\alpha}}\right)^{p} F^{p}(x)$ and $f^{p}(x)$ are effectively proportional. This would make a power of $x^{\alpha} / \alpha$, and then $\int f^{p}(x) d x$ would be divergent. Hence,

$$
\int_{0}^{\infty}\left(\frac{\alpha F(x)}{x^{\alpha}}\right)^{p} d_{\alpha} x \leq \frac{p}{p-1}\left\{\int_{0}^{\infty}\left(\frac{\alpha F(x)}{x^{\alpha}}\right)^{p} d_{\alpha} x\right\}^{p-1 / p}\left\{\int_{0}^{\infty} f^{p}(x) d_{\alpha} x\right\}^{1 / p} .
$$

Unless $f$ is nul. Since the integral on the left-hand side is positive and finite, (11) now follows from (15) as (13) followed from (12).

Using lemma 2.2 together with Theorem 2.1, we would like to introduce the following result.

Theorem 2.3 Let $f, g \geq 0, \alpha \in(0,1], F(x)=\int_{0}^{x^{\alpha} / \alpha} f\left(\alpha(t)^{\frac{1}{\alpha}}\right) d_{\alpha} t, G(y)=\int_{0}^{y^{\alpha} / \alpha} g\left(\alpha(t)^{\frac{1}{\alpha}}\right) d_{\alpha} t$, and assume that $0<\int_{0}^{\infty} f^{2}(x) d_{\alpha} x<\infty$, and $0<\int_{0}^{\infty} g^{2}(y) d_{\alpha} y<\infty$, then we have

$$
\begin{aligned}
\int_{0}^{\infty} \int_{0}^{\infty} & \frac{\alpha^{c+3}|\ln x-\ln y|^{c}}{a x^{\alpha}+b y^{\alpha}+\left|x^{\alpha}-y^{\alpha}\right|} \frac{F(x)}{x^{\alpha}} \frac{G(y)}{y^{\alpha}} d_{\alpha} x d_{\alpha} y \\
& \leq \mu\left(\int_{0}^{\infty} f^{2}(x) d_{\alpha} x\right)^{1 / 2}\left(\int_{0}^{\infty} g^{2}(y) d_{\alpha} y\right)^{1 / 2}
\end{aligned}
$$

Proof. Let

$$
I=\int_{0}^{\infty} \int_{0}^{\infty} \frac{\alpha^{c+3}|\ln x-\ln y|^{c}}{a x^{\alpha}+b y^{\alpha}+\left|x^{\alpha}-y^{\alpha}\right|} \frac{F(x)}{x^{\alpha}} \frac{G(y)}{y^{\alpha}} d_{\alpha} x d_{\alpha} y
$$

By Holder's inequality, we obtain

$$
\begin{aligned}
I \leq & \left\{\int_{0}^{\infty}\left(\int_{0}^{\infty} \frac{\alpha^{c+3}|\ln x-\ln y|^{c}}{a x^{\alpha}+b y^{\alpha}+\left|x^{\alpha}-y^{\alpha}\right|}\left(\frac{x}{y}\right)^{\alpha / 2} d_{\alpha} y\right)\left(\frac{F(x)}{x^{\alpha}}\right)^{2} d_{\alpha} x\right\}^{1 / 2} \\
& \times\left\{\int_{0}^{\infty}\left(\int_{0}^{\infty} \frac{\alpha^{c+3}|\ln x-\ln y|^{c}}{a x^{\alpha}+b y^{\alpha}+\left|x^{\alpha}-y^{\alpha}\right|}\left(\frac{y}{x}\right)^{\alpha / 2} d_{\alpha} x\right)\left(\frac{G(y)}{y^{\alpha}}\right)^{2} d_{\alpha} y\right\}^{1 / 2} .
\end{aligned}
$$


By using Lemma 2.1,

$$
\begin{aligned}
& I \leq\left\{\int_{0}^{\infty} \alpha^{2} A\left(\frac{F(x)}{x^{\alpha}}\right)^{2} d_{\alpha} x\right\}^{1 / 2} \times\left\{\int_{0}^{\infty} \alpha^{2} A\left(\frac{G(y)}{y^{\alpha}}\right)^{2} d_{\alpha} y\right\}^{1 / 2}, \\
& I \leq \alpha^{2} A\left\{\int_{0}^{\infty}\left(\frac{F(x)}{x^{\alpha}}\right)^{2} d_{\alpha} x\right\}^{1 / 2}\left\{\int_{0}^{\infty}\left(\frac{G(y)}{y^{\alpha}}\right)^{2} d_{\alpha} y\right\}^{1 / 2} .
\end{aligned}
$$

Finally, by Lemma 2.4 , for $p=2$, we have

$$
I \leq 4 A\left(\int_{0}^{\infty} f^{2}(x) d_{\alpha} x\right)^{1 / 2}\left(\int_{0}^{\infty} g^{2}(y) d_{\alpha} y\right)^{1 / 2} .
$$

Letting $\mu=4 A$, and inequality (16) is proved.

Corollary 2.1 Let $a=b=1$ in Theorem 2.3, then we obtain

$$
\begin{aligned}
\int_{0}^{\infty} \int_{0}^{\infty} \frac{\alpha^{c+3}|\ln x-\ln y|^{c}}{x^{\alpha}+y^{\alpha}+\left|x^{\alpha}-y^{\alpha}\right|} \frac{F(x)}{x^{\alpha}} \frac{G(y)}{y^{\alpha}} d_{\alpha} x d_{\alpha} y \\
\quad \leq K_{c}\left(\int_{0}^{\infty} f^{2}(x) d_{\alpha} x\right)^{1 / 2}\left(\int_{0}^{\infty} g^{2}(y) d_{\alpha} y\right)^{1 / 2}, \alpha \in(0,1]
\end{aligned}
$$

where the constant

$$
K_{c}=\alpha^{c} \int_{0}^{\alpha \frac{1}{\alpha}} 2^{c+1}|\alpha \ln h-\ln \alpha|^{c} d_{\alpha} h=2 c \alpha^{c} K_{c-1} . \quad \text { Here }, c=1,2,3, \ldots \text { and } K_{0}=2 .
$$

Proof: The proof of (17) is similar to that of (16), and here we only prove that:

$$
K_{c}=\alpha^{c} \int_{0}^{\alpha^{\frac{1}{\alpha}}} 2^{c+1}|\alpha \ln h-\ln \alpha|^{c} d_{\alpha} h=2 c \alpha^{c} K_{c-1}
$$

We have

$$
\begin{aligned}
K_{c} & =\int_{0}^{\infty} \frac{\alpha^{c+1}|\ln x-\ln y|^{c}}{x^{\alpha}+y^{\alpha}+\left|x^{\alpha}-y^{\alpha}\right|}\left(\frac{x}{y}\right)^{\alpha / 2} d_{\alpha} y \\
& =\int_{0}^{\infty} \frac{\alpha^{c+1}|\alpha \ln t-\ln \alpha|^{c}}{\alpha+t^{\alpha}+\left|\alpha-t^{\alpha}\right|}\left(\frac{\alpha}{t^{\alpha}}\right)^{1 / 2} d_{\alpha} t \\
& =\int_{0}^{\alpha^{\frac{1}{\alpha}}} \frac{\alpha^{c+1}|\ln t-\ln \alpha|^{c}}{2 \alpha}\left(\frac{\alpha}{t^{\alpha}}\right)^{1 / 2} d_{\alpha} t+\int_{\alpha}^{\infty} \frac{1}{\alpha} \frac{\alpha^{c+1}|\alpha \ln t-\ln \alpha|^{c}}{2 t^{\alpha}}\left(\frac{\alpha}{t^{\alpha}}\right)^{1 / 2} d_{\alpha} t .
\end{aligned}
$$

For the last integral, take $t^{\alpha}=\alpha^{2} s^{-\alpha}$ and rewrite this integral in term of $t^{\alpha}$, We obtain

$$
\begin{aligned}
K_{c} & =\int_{0}^{\alpha^{\frac{1}{\alpha}}} \frac{\alpha^{c+1}|\alpha \ln t-\ln \alpha|^{c}}{2 \alpha}\left(\frac{\alpha}{t^{\alpha}}\right)^{1 / 2} d_{\alpha} t+\int_{0}^{\alpha^{\frac{1}{\alpha}}} \frac{\alpha^{c+1}|\alpha \ln t-\ln \alpha|^{c}}{2 \alpha}\left(\frac{\alpha}{t^{\alpha}}\right)^{1 / 2} d_{\alpha} t \\
& =\int_{0}^{\alpha^{\frac{1}{\alpha}}} \alpha^{c}|\alpha \ln t-\ln \alpha|^{c}\left(\frac{\alpha}{t^{\alpha}}\right)^{1 / 2} d_{\alpha} t .
\end{aligned}
$$


Setting $h^{\alpha}=\left(\alpha t^{\alpha}\right)^{1 / 2}$, we get

$$
\begin{aligned}
K_{c} & =\int_{0}^{\alpha^{\frac{1}{\alpha}}} \alpha^{c}|\alpha \ln t-\ln \alpha|^{c}\left(\frac{\alpha}{t^{\alpha}}\right)^{1 / 2} d_{\alpha} t \\
& =\alpha^{c} \int_{0}^{\alpha^{\frac{1}{\alpha}}} 2^{c+1}|\alpha \ln h-\ln \alpha|^{c} d_{\alpha} h \\
& =2 c \alpha^{c} K_{c-1} .
\end{aligned}
$$

\section{$3 \quad$ Several Special Cases}

We now introduce some special inequalities of (16) by choosing different values for $a, b$, and $c$.

(1) If $a=c=0, b=1$, then we obtain

$$
\begin{aligned}
\int_{0}^{\infty} \int_{0}^{\infty} & \frac{\alpha^{3}}{y^{\alpha}+\left|x^{\alpha}-y^{\alpha}\right|} \frac{F(x)}{x^{\alpha}} \frac{G(y)}{y^{\alpha}} d_{\alpha} x d_{\alpha} y \\
& \leq \mu\left(\int_{0}^{\infty} f^{2}(x) d_{\alpha} x\right)^{1 / 2}\left(\int_{0}^{\infty} g^{2}(y) d_{\alpha} y\right)^{1 / 2},
\end{aligned}
$$

where $\mu=4 A$, and from Lemma 2.1,

$$
\begin{aligned}
A & =\int_{0}^{\alpha \frac{1}{\alpha}} \frac{2 \alpha^{2}}{\alpha^{2}} d_{\alpha} t+\int_{0}^{\alpha \frac{1}{\alpha}} \frac{2 \alpha^{2}}{2 \alpha^{2}-t^{2 \alpha}} d_{\alpha} t \\
& =\int_{0}^{\alpha \frac{1}{\alpha}} 2 d_{\alpha} t+2 \int_{0}^{\alpha \frac{1}{\alpha}}\left(\frac{1 / 2 \sqrt{2}}{\sqrt{2}-t^{\alpha} / \alpha}+\frac{1 / 2 \sqrt{2}}{\sqrt{2}+t^{\alpha} / \alpha}\right) d_{\alpha} t \\
& =2+\frac{1}{\sqrt{2}}\left[\ln \left(\frac{\sqrt{2}+t^{\alpha} / \alpha}{\sqrt{2}-t^{\alpha} / \alpha}\right)\right]_{0}^{\frac{1}{\alpha}} \\
& =3.24646 .
\end{aligned}
$$

(2) If $a=1, b=1, c=0$, then

$$
\begin{aligned}
\int_{0}^{\infty} \int_{0}^{\infty} & \frac{\alpha^{3}}{x^{\alpha}+2 y^{\alpha}+\left|x^{\alpha}-y^{\alpha}\right|} \frac{F(x)}{x^{\alpha}} \frac{G(y)}{y^{\alpha}} d_{\alpha} x d_{\alpha} y \\
& \leq \mu\left(\int_{0}^{\infty} f^{2}(x) d_{\alpha} x\right)^{1 / 2}\left(\int_{0}^{\infty} g^{2}(y) d_{\alpha} y\right)^{1 / 2}
\end{aligned}
$$

where $\mu=4 A$, and from Lemma 2.1,

$$
\begin{aligned}
A & =\int_{0}^{\alpha \frac{1}{\alpha}} \frac{2 \alpha^{2}}{2 \alpha^{2}+t^{2 \alpha}} d_{\alpha} t+\int_{0}^{\alpha \frac{1}{\alpha}} \frac{2 \alpha^{2}}{3 \alpha^{2}} d_{\alpha} t \\
& =\sqrt{2}\left[\tan ^{-1} \frac{1}{\sqrt{2}}-\tan ^{-1} 0\right]+\frac{2}{3} \\
& =2.2071 .
\end{aligned}
$$


(3) If $a=b=0, c=1$, then

$$
\begin{aligned}
\int_{0}^{\infty} \int_{0}^{\infty} \frac{\alpha^{4}|\ln x-\ln y|}{\left|x^{\alpha}-y^{\alpha}\right|} \frac{F(x)}{x^{\alpha}} \frac{G(y)}{y^{\alpha}} d_{\alpha} x d_{\alpha} y \\
\quad \leq \mu\left(\int_{0}^{\infty} f^{2}(x) d_{\alpha} x\right)^{1 / 2}\left(\int_{0}^{\infty} g^{2}(y) d_{\alpha} y\right)^{1 / 2},
\end{aligned}
$$

where $\mu=4 A$, and from Lemma 2.1,

$$
\begin{aligned}
A & =8 \int_{0}^{\alpha^{\frac{1}{\alpha}}} \frac{\alpha^{2}\left|\ln \left(\frac{t^{\alpha}}{\alpha}\right)\right|}{\alpha^{2}-t^{2 \alpha}} d_{\alpha} t \\
& =-8 \alpha^{2} \int_{0}^{\alpha^{\frac{1}{\alpha}}} \frac{\ln \left(\frac{t^{\alpha}}{\alpha}\right)}{\alpha^{2}-t^{2 \alpha}} d_{\alpha} t \\
& =-8\left[\sum_{k=1}^{\infty} \frac{\left(1-\frac{t^{\alpha}}{\alpha}\right)^{k}}{k^{2}}+\sum_{k=1}^{\infty} \frac{\left(-\frac{t^{\alpha}}{\alpha}\right)^{k}}{k^{2}}+\ln \left(\frac{t^{\alpha}}{\alpha}\right) \ln \left(\frac{t^{\alpha}}{\alpha}+1\right)\right]_{0}^{\alpha^{\frac{1}{\alpha}}} \\
& =8\left[\sum_{k=1}^{\infty} \frac{1}{k^{2}}-\sum_{k=1}^{\infty} \frac{(-1)^{k}}{k^{2}}\right] \\
& =16 \sum_{k=1}^{\infty} \frac{1}{(2 k-1)^{2}} \\
& =2 \pi^{2} .
\end{aligned}
$$

(4) If $a=c=0, b=2$, then

$$
\begin{aligned}
\int_{0}^{\infty} \int_{0}^{\infty} \frac{\alpha^{3}}{2 y^{\alpha}+\left|x^{\alpha}-y^{\alpha}\right|} \frac{F(x)}{x^{\alpha}} \frac{G(y)}{y^{\alpha}} d_{\alpha} x d_{\alpha} y \\
\quad \leq \mu\left(\int_{0}^{\infty} f^{2}(x) d_{\alpha} x\right)^{1 / 2}\left(\int_{0}^{\infty} g^{2}(y) d_{\alpha} y\right)^{1 / 2}
\end{aligned}
$$

where $\mu=4 A$, and from Lemma 2.1,

$$
\begin{aligned}
A & =\int_{0}^{\alpha \frac{1}{\alpha}} \frac{2 \alpha^{2}}{\alpha^{2}+t^{2 \alpha}} d_{\alpha} t+\int_{0}^{\alpha \frac{1}{\alpha}} \frac{2 \alpha^{2}}{3 \alpha^{2}-t^{2 \alpha}} d_{\alpha} t \\
& =\left.2 \tan ^{-1}\left(\frac{t^{\alpha}}{\alpha}\right)\right|_{0} ^{\frac{1}{\alpha}}+\left.\frac{1}{\sqrt{3}}\left(\ln \left(\sqrt{3}+\frac{t^{\alpha}}{\alpha}\right)-\ln \left(\sqrt{3} \frac{t^{\alpha}}{\alpha}\right)\right)\right|_{0} ^{\alpha^{\frac{1}{\alpha}}} \\
& =\frac{\pi}{2}+\frac{1}{\sqrt{3}}(\ln (\sqrt{3}+1)-\ln (\sqrt{3}-1)) \\
& =2.3311423 .
\end{aligned}
$$

\section{Remark 3.1} [2].

In the previous special inequalities, if we take $\alpha=1$, then we get the same results that obtained by Abu Hany 


\section{References}

[1] A.A.K. Abu Hany, M.S. El-Khatib, K.A.K. Shourrab, Some New HilbertType Fractional Inequalities, IUG Journal of Natural Studies, 27(1) 2019, 44-52 .

[2] A.A.K. Abu Hany, On Some Integral Analogues to Hilbert's inequality, Gen. Math. Notes, 20 (1) $2014,58-66$.

[3] G.H. Hardy, Note on a theorem of Hilbert, Mathematische Zeitschrift, 6(3-4) 1920, 314-317. https://doi.org/10.1007/bf01199965

[4] H.X. Du, Y. Miao, Several analogues of Hilbert inequalities, Demonstratio Math.XLII (2) (2009), 297302.

[5] K. Mario, P. Josip, General Hilbert's and hardy's Inequalities, Mathematical inequalities and Applications, 8 (1)2005, 29-51.

[6] U.N. Katugampola, A new fractional derivative with classical properties, J. American Math. Soc., arXiv : $1410.6535 \mathrm{v} 2$ (2014).

[7] W.T. Sulaiman, Four inequalities similar to Hardy-Hilbert's integral inequality, J. Inequal. Pure Appl. Math (JIPAM), 7(2) 2006 (Article 76).

[8] Y. Li, J. Wu, B. He, A new Hilbert-type integral inequality and the equivalent from, International Journal of Mathematics and Mathematical Sciences, 2006, Article ID 45378, 6 pages, 2006.https://doi.org/10.1155/ijmms/2006/45378

[9] Y. Li, Y. Qian, B. He, On Further Analogs of Hilbert's Inequality, International Journal of Mathematic and Mathematical Sciences, vol. 2007, Article ID 76329, 6 pages, 2007.https://doi.org/10.1155/2007/76329

[10] Y. Miao, H. X. Du, A note on Hilberttype integral inequality.Inequality Theory and Applications. 6 (2010), 261-267. 